\title{
MANEJO FARMACOLÓGICO DE DOLOR OROFACIAL. REVISIÓN
}

\author{
Aleida Neira Odontóloga ${ }^{i^{*}}$, Andrés Olaya Md., Toxicólogo Clinicoª \\ ${ }^{1}$ Residente del Programa de Cirugía Oral y Maxilofacial, Universidad Militar Nueva Granada, Bogotá, D.C. Co- \\ lombia ${ }^{2}$ Toxicólogo Clínico, Universidad de Antioquia, Residente del Programa de Farmacología Clínica U de la \\ Sabana, Docente de Cátedra Facultad de Medicina Universidad Militar Nueva Granada, Bogotá D.C. Colombia.
}

\begin{abstract}
Resumen
El dolor orofacial en sus fases aguda y crónica, sea somático de etiología neuropática, o asociado a condiciones psicológicas, tiene una alta prevalencia en nuestro medio. Múltiples opciones terapéuticas de tipo farmacológico se convierten en la primera línea de tratamiento y la correcta elección depende de la patología subyacente. Esta decisión no siempre se facilita debido a que no existe unidad en cuanto a los criterios diagnósticos para su clasificación. Teniendo en cuenta la multiplicidad de opciones, el objetivo de la presente revisión es analizar los tratamientos descritos en la literatura científica actual, con el propósito de brindar elementos que faciliten tomar una decisión certera en cada caso específico de dolor orofacial.
\end{abstract}

Palabras clave: dolor orofacial, neuralgia del trigémino, dolor crónico

\section{PHARMACOLOGIC MANAGEMENT OF OROFACIAL PAIN. REVIEW}

\begin{abstract}
Orofacial pain in its acute and chronic phases, either of somatic etiology or associated with psychological conditions is of highly prevalence. Multiple pharmacological therapies are the first line of treatment, and the correct choice depends on the underlying pathology. This decision is not always easy because there is not unity in the diagnostic criteria used for its classification. Given the multiplicity of options, the objective of this review is to analyze the treatments described in the current scientific literature, in order to provide the elements to make an accurate decision in each specific case of orofacial pain.
\end{abstract}

Keywords: orofacial pain, trigeminal neuralgia, chronic pain

\section{TRATAMENTO FARMACOLÓGICO DA DOR OROFACIAL. REVISÃO}

\section{Resumo}

\begin{abstract}
A dor orofacial nas fases aguda e crônica, seja somática de etiología neuropática ou associada a condições psicológicas, é altamente prevalente em nosso meio. Várias opções terapéuticas de tratamento farmacológico se tornarem a primeira linha de tratamento ea escolha correta depende da patologia de base. Esta decisão nem sempre é fácil porque não há nenhuma unidade em termos de critérios diagnósticos para a classificação. Dada a multiplicidade de opções, o objetivo desta revisão é analisar os tratamentos descritos na literatura científica atual, a fim de fornecer elementos que facilitem tomar uma decisão correta em cada caso específico da dor orofacial.
\end{abstract}

Palabras-chave: dor facial, neuralgia do trigêmeo, dor crônico

* Correspondencia: Aleida Neira alenei96@yahoo.es. Dirección postal: Servicio de Cirugía Oral y Maxilofacial, Tr. 3 \# 49-00 Hospital Militar Central, Bogotá D.C., Colombia.

Recibido: Diciembre 28 de 2009 Aceptado: Junio 2 de 2010. 


\section{Introducción}

El dolor orofacial se presenta en todos los grupos etarios y se reconoce fácilmente cuando su origen es dental, como sucede en los casos de caries y de enfermedad periodontal. Igualmente, un alto porcentaje de individuos que padecen problemas de articulación temporo-mandibular lo identifican, por los característicos síntomas de crepitación articular, dolor de la musculatura masticatoria, dolor articular, limitación a la apertura oral, cefalea, dolor referido al área sinusal y algunos síntomas otológicos. Estudios epidemiológicos describen que alrededor de un $33 \%$ de la población sana tiene algún tipo de alteración temporo-mandibular y que entre el $30 \%$ y el $60 \%$ se manifiesta con algún tipo de sintomatología, estimándose que únicamente el $5 \%$ requiere de algún tipo de tratamiento (1). El síndrome de disfunción temporo-mandibular (SDTM) aparece más frecuentemente hacia la tercera década de vida, seguida por la cuarta y segunda respectivamente. Se ha observado predominio en el sexo femenino, con una relación de géneros que varía en las diferentes publicaciones, acercándose todas a la relación 2:1 $(1,2)$. Para algunos autores el SDTM es la causa más común de dolor crónico localizado en la región orofacial, calculando que un $12 \%$ de población es afectada (3). También se ha observado en el género masculino una propensión a la sensibilidad relacionada con las variaciones de temperatura (4).

Dentro de las etiologías de dolor orofacial se debe resaltar la neuralgia del trigémino, un trastorno de localización típicamente unilateral en el $96 \%$ de los casos, que se presenta con más frecuencia entre la quinta y la séptima década, con una sutil predilección por el sexo femenino $(1,74: 1)$ y una tasa de incidencia anual de entre cuatro y cinco por cada cien mil habitantes (3). También se puede presentar dolor orofacial secundario a la quimioterapia y a la radioterapia en pacientes con cáncer y por alteraciones musculares $e$ inmunológicas que requieren tratamiento paliativo y analgésico (5). Como el diagnóstico del dolor orofacial se dificulta por su etiología multifactorial, es indispensable elaborar una minuciosa y detallada historia clínica y anamnesis, a fin de identificar el máximo de factores que de manera cotidiana afectan la salud del paciente. Seguidamente se debe realizar un completo análisis del macizo facial, estableciendo escalas de dolor y haciendo una certera exploración física que incluya palpación muscular y auscultación de la articulación temporo-mandibular, criterios estos que junto con radiografías y resonancias magnéticas con técnicas de boca abierta y cerrada, facilitan establecer la causa de la patología y orientar su manejo (6).

\section{Clasificación y definiciones para el dolor orofacial}

En 1988, la Sociedad Internacional de Cefalea publicó la primera clasificación y criterios de diagnósticos para los trastornos de dolor de cabeza, las neuralgias craneales y el dolor facial, la cual se reevaluó en el 2004 con base en las estructuras responsables de producir el estímulo nociceptivo (verdadero origen del dolor y eje que fisiológicamente influye en la experiencia del dolor). Se propuso el sistema de ejes (I y II) como factores que influencian la intensidad de dolor, en donde el eje I relaciona los dolores agudos, evalúa los aspectos físicos y tiene buena respuesta a terapias dirigidas a la entrada somato sensorial. Los dolores crónicos en cambio tienen importante relación con el Eje II, el cual evalúa los aspectos psicológicos, psicosociales y la incapacidad orofacial, se caracteriza por una pobre respuesta al tratamiento a nivel somato sensorial (7) y requiere de tratamiento psicológico que ayude en su manejo (8). Jeffrey Okeson propuso la clasificación que se describe a continuación para del dolor orofacial agudo relacionado con el Eje I: somático superficial (dolor cutáneo y mucogingival), somático profundo (músculo-esquelético y visceral) y neuropático (periférico y central) (7).

\section{Dolores somáticos}

Dolor músculo-esquelético: dolor muscular, dolor local, miofacial, mioespasmo, miositis, mialgias.

Dolor de articulación temporomandibular: de ligamentos, retrodiscal, capsular, dolor artrítico, dolor óseo, perióstico, de tejido conectivo, dental y periodontal.

Dolor miofascial: presencia de centros de coordinación, zonas musculares con áreas de hipersensibilidad llamados puntos gatillo que producen un dolor exquisito (3,7,9-11), asociado frecuentemente con dolor lumbar por dolor referido $(11,12)$.

Miositis: inflamación muscular aguda por infección o por traumatismo $(3,7)$.

Mioespasmo: caracterizado por dolor muscular fuerte agudo y limitación marcada de apertura oral, a menudo con maloclusión $(3,7)$ 
Mialgias locales: dolor muscular espontáneo, sensibilidad a la palpación muscular, apertura oral limitada y síntomas que se agravan con actividades que requieren movimiento como masticar, hablar, reír o bostezar $(3,7)$.

Alteraciones disco articular: caracterizadas por desplazamiento del disco articular hacia anterior y hacia dentro. Las alteraciones en el disco-cóndilo y la relación estructural puede ser consecuencia de elongación de los ligamentos discales secundaria a trauma. Altos niveles de ansiedad y de bruxismo son factores de riesgo para el inicio de alteraciones del disco $(3,7)$. El trastorno de la articulación ocasiona el mayor porcentaje de dolor facial (13).

Sinovitis y capsulitis: inflamación del revestimiento sinovial de la articulación temporo-mandibular y del ligamento capsular que causa profundo dolor, sensibilidad a la palpación, limitación a la apertura oral secundaria al dolor e inadecuada oclusión de los dientes posteriores en el lado afectado $(3,7$, 14,15).

Artrosis: condición no inflamatoria de artritis con deterioro de las superficies articulares. Se presenta con dolor agravado por el movimiento de la mandíbula, dolor a la palpación de la articulación, crepitación y limitación al movimiento $(3,7,14,15)$.

Dolor visceral pulpar glandular: de fácil localización por el paciente, su etiología es multifactorial y su tratamiento consiste la eliminación del agente causal seguida de antibioticoterapia $(16,17)$

\section{Dolores neurovasculares}

Cefalea de tipo tensional (ETTH): dolor de cabeza primario que afecta las zonas occipital, parietal, temporal o frontal, usualmente bilateral, de intensidad leve a moderada, con una duración de pocas horas a siete días. Contrario a la migraña, no cursa con náuseas o vómitos y no hay empeoramiento del dolor con la actividad física de rutina (13,16-18).

Cefalea tensional crónica (CTTH): similar a la ETTH, se caracteriza por dolor de cabeza por más de quince días al mes, durando hasta tres meses. Por lo general evoluciona de una ETTH y puede asociarse a fotofobia, fonofobia o leves náuseas (13,16-18).

\section{Dolores neuropáticos}

Neuralgia del trigémino (TN): se caracteriza por dolor episódico, lacinante en la distribución de una o más divisiones del nervio trigémino $(3,19)$. Para el diagnóstico se reconocen dos subgrupos: TN clásica (antes idiopática o primaria), relacionada con la compresión neurovascular y TN secundaria, asociada a otras patologías. El dolor de la musculatura de la cara (tic doloroso), es intermitente, paroxístico, localizado en media cara, inicialmente afectando una sola de las tres ramas trigeminales (rara en la primera rama), sin causa evidente, de aparición brusca y espontánea, provocado por el menor estímulo sobre uno de los llamados puntos gatillo (16), que se localizan en cejas (primera rama), punto infraorbitario, labio superior y ala nasal (segunda rama) y labio inferior, encía y mentón (tercera rama). Una característica importante que distingue el TN del dolor dental es que en aquel no se suele interrumpir el sueño del paciente, mientras que en el originado por patología dental si, debido a su carácter generalmente progresivo que cambia con el tiempo (16). La neuralgia del trigémino se puede desarrollar secundaria a lesiones de fosa posterior, a tumores intracraneales, los cuales se detectan en un $2 \%$ de los pacientes. También por múltiples placas de desmielinización, con la participación de esclerosis nociceptivas del trigémino (14, 19-21).

Neuralgia del glosofaríngeo: de naturaleza idiopática, se caracteriza por dolor intenso de aparición brusca en la región amigdalina y lingual posterior, irradiado hacia distintas zonas, especialmente al oído. Se hace más intenso con la deglución, es muy similar a la neuralgia del trigémino, apareciendo con más frecuencia de la sexta a octava década $(16,17,22)$.

Neuralgia post-herpética (NPH): complicación aguda del herpes zóster relativamente común, puede producirse décadas después de la infección primaria (varicela). Se trata de una enfermedad de los ganglios de la raíz dorsal que induce una erupción vesicular (dermatoma del trigémino). La rama oftálmica se ve afectada en más del $80 \%$ de los casos, especialmente en varones de edad avanzada $(22,23)$.

Neuralgia traumática: ocurre después de una lesión neural directa, en ocasiones con retraso en la aparición de dolor después de la lesión (22).

Síndrome de Eagle: rara condición más frecuente en mujeres, que resulta de la compresión del nervio glo- 
sofaríngeo por una apófisis estiloides alargada, o por el ligamento estilo-hioideo osificado. Los principales signos y síntomas incluyen dolor sordo persistente, dolor de garganta, disfagia, otalgia y sensación de cuerpo extraño (22).

Fibromialgias: es un cuadro de dolor crónico benigno, generalizado y de etiología desconocida, referido a diversas estructuras del sistema músculo-esquelético $(22,24,25)$. Cursa con trastornos reumatológicos inespecíficos, presente durante tres meses o más, con respuesta aguda a la palpación digital en por lo menos once de los dieciocho puntos sensibles examinados bilateralmente: occipital, cervical, trapecio, supraespinoso, segunda costilla, epicóndilolateral, glúteos, trocánter mayor y rodilla. Afecta del $2 \%$ al $4 \%$ de la población y se acompaña de trastornos del sueño, rigidez, fatiga, depresión y ansiedad (26-30).

Se resalta la importancia de establecer las diferencias entre fibromialgia y dolor miofascial. La fibromialgia presenta características como dolor diseminado, la sensibilización es central y si el dolor es local tiene un punto sensible y punto gatillo. Además, hay dolor músculo-esquelético y la rigidez es generalizada $e$ irreversible. En cambio en el dolor miofascial, el dolor es regional, los puntos gatillo ayudan al diagnóstico, la sensibilización es periférica, el dolor es referido de puntos gatillo, se afecta el vientre muscular y hay respuesta al tratamiento (31).

\section{Medicamentos utilizados en el tratamiento del dolor orofacial}

\section{Analgésicos opioides}

Los opioides son sustancias de origen natural y sintético que interactúan con receptores acoplados a proteínas Gi (inhibitorias) en neuronas localizadas a nivel central y periférico; hacen parte de un sistema corporal especializado que media la transmisión y percepción de los estímulos dolorosos proporcionando analgesia y son tradicionalmente usados para el manejo del dolor agudo y crónico. Los efectos adversos mas importantes incluyen sedación, depresión respiratoria, nauseas, estreñimiento y dependencia, la mayoría de estos relacionados con la dosificación y el periodo de administración de los mismos (32-35).

El tramadol es un fármaco que se usa solo, o en terapia combinada con el acetaminofén (36). Además de ser agonista del receptor $\mu$, interactúa con receptores de serotonina y norepinefrina creando un efecto analgésico con dosis de mantenimiento de 300 a $400 \mathrm{mg} /$ día en cuatro dosis y de 2 a $3 \mathrm{~g} /$ día en tres dosis de acetaminofén En un estudio multicéntrico realizado en el 2003 con tramadol (37,5 mg) y acetaminofén (325 mg) en tabletas combinadas para el tratamiento de fibromialgia, se observó mejoría del dolor en el 75,6\% de los pacientes, n:416 (37). Es así como se considera una buena alternativa en la terapia antiálgica, debido a su efectiva actividad analgésica en los síndromes de dolor crónico, utilizándose de forma segura con un mínimo riesgo (38). En dosis de 50 a 100 mg tres veces al día es importante en la terapia de fibromialgia (39).

\section{Antinflamatorios no esteroideos (AINES)}

Los AINES son fármacos que inhiben la acción de la enzima ciclooxigenasa en sus diferentes isoformas, COX-1, COX-2 y COX-3, disminuyendo la producción de prostaglandinas y tromboxanos, eventos que reducen la nocicepción (40). El uso de estos medicamentos se asocia con muchos beneficios, dentro de ellos la disminución en el uso de opioides ("ahorradores de opioides"), que lleva a una disminución de efectos colaterales como náusea, vómito, estreñimiento y sedación (41). Los AINES tradicionales están limitados por los efectos adversos relacionados con la dosis, por la cronicidad del tratamiento y en casos de patologías preexistentes como la enfermedad ulcero péptica, la disfunción plaquetaria y la insuficiencia renal, entre otras (32). Hay que recordar que mientras la COX-1 es una enzima constitutiva en casi todos los tejidos, en especial del riñón y del sistema digestivo (lo que explica la aparición de los efectos adversos durante su inhibición), la COX-2 es una enzima por excelencia inducible ante agresión (mediadora del dolor e inflamación), pero que a su vez cuenta con una fracción constitutiva en algunos órganos vitales como riñón y el endotelio, lo que explica los efectos adversos relacionados con el riesgo cardiovascular cuando se inhibe (42). Algunos autores recomiendan el uso de este grupo de medicamentos en el dolor orofacial (43). Es así como la decisión de usar AINES sistémicos en dolores musculares como monoterapias, o en dolores crónicos, se debe evaluar con relación a la sinergia que pueden presentar con otros medicamentos $(3,14,15,44)$.

El acetaminofén, un analgésico perteneciente al grupo de los AINEs derivado del acido para-aminofenol, ha demostrado en numerosos estudios sinergia con anal- 
gésicos de tipo opioide en adultos, en dosis de cuatro gramos diarios divididos en cuatro dosis, teniendo precaución en pacientes alcohólicos, desnutridos, o con disfunción hepática. Posee también un efecto potencial en la terapia multimodal (combinación de varias clases de fármacos e intervenciones terapéuticas) (32). El diclofenac, medicamento derivado del acido acético, pertenece al grupo de los AINEs no selectivos (COX1 y COX2), proveyendo un buen control del dolor postoperatorio y también disminución del requerimiento de opioides. En un estudio comparativo se mostró que la eficacia del diclofenac sódico era similar a la del tramadol, por lo que su administración postoperatoria en cirugía ortognática se acepta para el control del dolor (36). En otro estudio que incluyó 119 pacientes a quienes se les realizó cirugía ambulatoria bajo anestesia para extracción de terceros molares impactados, no se observaron diferencias significativas entre el efecto del ibuprofeno $600 \mathrm{mg}$, el diclofenaco $100 \mathrm{mg}$ y el paracetamol $1 \mathrm{~g}$ con $60 \mathrm{mg}$ de codeína en el control del dolor postoperatorio del dolor (45).

Desde 1978 se viene observando en la literatura científica mundial que la administración de aspirina (ácidoacetil-salicílico) previa a las intervenciones quirúrgicas, reduce significativamente la respuesta de agregación plaquetaria, aumentado de manera importante el sangrado durante y después de la cirugía, la incidencia de hematomas, la equimosis y el edema de manera subjetiva. De ahí que los AINES, como medicamentos preventivos para el dolor o "analgesia anticipada" se usen de manera eficaz al mostrar eficacia antiinflamatoria durante el posoperatorio, reduciendo también en los adultos el consumo global de narcóticos y sus efectos adversos. Entre ellos el ibuprofeno ha sido el más estudiado y documentado (46). Vale la pena mencionar que existen estudios que aseveran que los métodos tradicionales de control del dolor con AINEs en el tratamiento de trastornos degenerativos e inflamatorios músculo-esqueléticos como es la fibromialgia, no resulta muy eficaz (47).

\section{Toxina botulínica}

Utilizada para el manejo del mioespasmo, la toxina botulínica de tipo A previene la unión de la acetilcolina en las terminales presinápticas neuromusculares, mostrando ser efectiva en distonías y mialgias de cabeza y cuello, así como disfunciones de la articulación temporo-mandibular; confiere también mejoría de los síntomas durante aproximadamente cuatro meses, que corresponde a su tiempo de actividad. Como desventaja, algunos autores consideran que se trata de un tratamiento temporal y bastante costoso $(11,48)$. Hay estudios que sugieren que podría tener efectos analgésicos, al parecer mediados por la inhibición en la liberación de glutamato y por la reducción en la producción de sustancia $\mathrm{P}$ (péptido amplificador del glutamato) (31). La toxina de tipo A ha tenido un uso clínico más extenso y generalizado que la de tipo $\mathrm{B}$, mostrando eficacia en el tratamiento de distonía cervical, en espasticidad y en las líneas de hipercinéticos faciales. En la actualidad se está proponiendo como tratamiento para dolor miofascial y para algunos tipos de cefalea (49), con un efecto positivo entre las cuatro y las seis semanas después de la aplicación (50). También se viene usando en tics faciales, en discinesias orofaciales y en distonía e hipertrofia idiopática de los músculos masticadores (51). Mientras que su eficacia se ha demostrado en los síndromes dolorosos asociados a espasticidad, en los que existe un incremento de la actividad muscular, en cefalea tensional su actividad es limitada (31).

\section{Antidepresivos tricíclicos}

Estos fármacos, indicados en el tratamiento de la depresión, han mostrado efectos benéficos en dosis usualmente bajas, en el manejo de algunos tipos de dolor incluyendo el dolor miofascial $(3,52)$. Poseen múltiples mecanismos de acción relacionados con el transporte de neurotransmisores (norepinefrina, serotonina), bloqueo de receptores (histamina, acetilcolina) y transporte de iones (Na y K). También han mostrado utilidad en la cefalea tensional crónica $(16,17)$. El más utilizado ha sido la amitriptilina en fibromialgias, recomendándose dosis de $10-25 \mathrm{mg}$ en horas nocturnas, a fin de mejorar el patrón de sueño y el efecto analgésico $(14,20,24,53)$. En neuralgias posherpéticas se ha usado en dosis de entre 12,5 a $25 \mathrm{mg} /$ día, con un máximo de $250 \mathrm{mg} /$ día. En términos generales, su rango de dosis efectiva es de 12,5 a $150 \mathrm{mg}$ diarios, con una dosis promedio es de $70 \mathrm{~g} /$ día (23). Mediante el bloqueo de la recaptación de noradrenalina y de serotonina en el asta dorsal de la medula, suelen ser eficaces en la hipersensibilidad neuropática y central, con la desventaja de algunos efectos secundarios a su poder antihistamínico y anticolinérgico que causa sedación, boca seca, estreñimiento, taquicardia, arritmias y ortostatismo (20). Aunque la amitriptilina ha sido el tricíclico más estudiado, las aminas secundarias del mismo grupo pueden ser igualmente eficaces, 
ocasionando menores efectos secundarios. Después de descartar significativamente las contraindicaciones cardiacas, la amitriptilina se puede iniciar de 10 a 25 $\mathrm{mg}$ cada noche, incrementando $50 \mathrm{mg}$ después de una semana de terapia, teniendo en cuenta que sus propiedades analgésicas toman entre tres y cuatro semanas. En un estudio con pacientes post-amputados se observó que más del $80 \%$ presentaba alivio con un promedio de $56 \mathrm{mg}$ de amitriptilina por día (47) y en un metanálisis que analizó la eficacia de antidepresivos en el dolor de la fibromialgia se encontró que mostraban más beneficios que los inhibidores selectivos de recaptación de serotonina (ISRS) en el tratamiento del dolor neuropático, encontrándose también que la combinación de tricíclicos con inhibidores selectivos de la recaptación de serotonina (ISRS) era más eficaz que cuando se suministraban como medicamentos únicos (47).

\section{Benzodiacepinas}

En muchos pacientes con trauma se incrementa la ansiedad, la ira, la frustración y el estrés, no solo por sus heridas, sino por el impacto profundo en su vida familiar. En esos casos las benzodiacepinas, que pertenecen al grupo de los ansiolíticos y que son agonistas del receptor del GABAA (ácido-gamino-butírico) producen una respuesta clínica significativa en el paciente politraumatizado, disminuyendo el dolor, la sedación y la ansiolisis. En pacientes que reciben repetidos procedimientos en las unidades de cuidado intensivo se ha demostrado beneficio tras el uso de ansiolíticos (32). En pacientes con neuralgia traumática las benzodiacepinas han logrado controlar el dolor en el $40 \%$ de los casos, con dosis de 6-8 mg/día, al aumentar el influjo del ión cloro se lleva a una hiperpolarización de las neuronas (54). También hay reportes en que los antidepresivos tricíclicos, los suplementos nutricionales, las benzodiacepinas, los inhibidores selectivos de serotonina, los analgésicos no esteroides, los corticoides y la combinación de relajantes musculares, se han utilizado en el manejo de la disfunción y del dolor muscular masticatorio (20).

\section{Anticonvulsivantes}

Estos medicamentos se vienen utilizando desde hace más de cincuenta años en el manejo del dolor neuropático crónico y se considera que su efectividad puede llegar hasta el 95\%. Han jugado un papel importante en el tratamiento de la neuropatía diabética periférica y de la neuralgia postherpética (16). El gabapentín la pregabalina, análogos estructurales de la g-amino-butírico, reducen como tal la entrada de calcio en los canales de calcio, incrementando la actividad no adrenérgica y reduciendo en la médula espinal la excitación de aminoácidos, glutamato y aspartato. La pregabalina tiene un perfil farmacocinético favorable que permite una acción terapéutica más rápida y una dosificación dos veces al día. Se inicia a $300 \mathrm{mg}$, tres veces al día, aumentando $300 \mathrm{mg}$ por dosis cada tres días, hasta un máximo de dosis diaria de 3,6 g. Los más frecuentes efectos secundarios son mareo y somnolencia en un $10 \%$ de los pacientes, siendo muy bien tolerada en la mayoría de ellos. La gabapentina se ha usado en varios estudios en el tratamiento del dolor de miembro fantasma y su beneficios incluyen analgesia, disminución promedio de $50 \%$ menos de opiáceos, de ansiedad y del dolor crónico $(16,20,24,32,55)$.

La carbamazepina (CBZ) es el pilar de la farmacoterapia para la neuralgia del trigémino (56) y la primera elección para el manejo de esta patología. Aunque la monoterapia con ella controla los síntomas iníciales hasta un $80 \%$ de los casos, también se ha utilizado oxcarbazepina, Iamotrigina (LTG), fenitoína, gabapentina y tizanidine con evidencia de alta efectividad. A menudo las combinaciones de medicamentos se utilizan para maximizar la eficacia y minimizar los efectos negativos (19). Las dosis utilizadas van desde 100 a $2400 \mathrm{mg}$ al día, con un importante porcentaje de pacientes que responden con 200 a $800 \mathrm{mg}$ al día, divididos en dos o tres dosis diarias (19). En casos sin respuesta a la carbamazepina, la fenitoina se convierte en el fármaco de elección y si la presencia de efectos secundarios limita el uso de los anticonvulsivantes, se puede utilizar baclofeno (BCF), un relajante muscular agonista del ácido g-amino- aminobutírico (56). El uso de fenitoina para la neuralgia del trigémino data desde $1942(21,56)$, pero la mayoría de estudios muestran que la $\mathrm{CBZ}$, el BCF y la LTG pueden ser superiores a ella, con menos efectos secundarios. El BCF es una opción alternativa para el tratamiento inicial y sus propiedades neurofarmacológicas, su eficacia y seguridad las documentó cuidadosamente Fromm por más de dos décadas. La experiencia de muchos estudios es que BCF puede ser tan eficaz como CBZ y a menudo mejor tolerado en casos de neuralgia del trigémino (56). La administración de BCF es intratecal y en distonías faciales es mucho más eficaz que cuando se administra por vía oral (51). 
Cuando el paciente no responde a un solo medicamento se recomienda agregar un segundo anticonvulsivante para mejorar la respuesta terapéutica. Scrivani encontró que mientras el $50 \%$ de los pacientes tenía un alivio satisfactorio del dolor con un sólo medicamento, el $70 \%$ reportaba una respuesta satisfactoria con dos fármacos (21). En fibromialgias, en donde el tratamiento farmacológico es multifactorial, los anticonvulsivantes se han usado con resultados favorables (37). Los antidepresivos tricíclicos y los inhibidores selectivos de la recaptación de serotonina se consideran los más eficaces y seguros, en particular el duloxetine (IRSN), a dosis de 30 a $60 \mathrm{mg}$ dos veces al día, mucho más seguro que el TCA en el tratamiento prolongado. La gabapentina también ha mostrado ser eficaz en el alivio del dolor en un $50 \%$ de los pacientes, disminuyendo la frecuencia de dormir y aliviando los demás síntomas (39).

\section{Relajantes musculares}

Se han utilizado para el manejo del síndrome miofascial y de mioespasmos con compromiso de la musculatura a nivel cervical y lumbar. La tizanidina, un agonista a-2-adrenérgico derivado de la imidazolina es uno de los más usados, en dosis de 2 a 4 o mg, con incremento de dos mg si es necesario, para un máximo de $36 \mathrm{mg}$ día. El metocarbamol, carbamato análogo derivado del mefenesín, produce inhibición de los reflejos polisinápticos y se suministra en tabletas de 500 a $750 \mathrm{mg}$, con dosis usuales en adultos es de un gramo cuatro veces al día $(31,54)$.

\section{Conclusiones}

El adecuado manejo terapéutico del paciente con dolor orofacial depende de un correcto diagnóstico, siendo necesario para ello una rigurosa historia clínica, que debe incluir máximo de antecedentes fisiológicos, patológicos, psicológicos y ambientales, esenciales la identificación de los factores propuestos para los ejes I y II, determinantes en la conducta a seguir. A ello se suma la importancia de contar con unas adecuadas ayudas diagnósticas, indispensables para confirmar la etiología del dolor, que como se ha descrito, responde a múltiples causas. Es importante tener en cuenta el género, porque está descrito que el sexo femenino presenta mayor factor de riesgo para dolor de articulación temporo-mandibular y dolor facial, en contraste con el género masculino, más propenso a reportar sensibilidad con la temperatura. Al involucrar el dolor orofacial en un heterogéneo grupo de enfermedades, su tratamiento exige ser multidisciplinario. Se resalta la importancia de establecer las diferencias entre fibromialgia y dolor miofascial.

El primer tratamiento a considerar deben ser las terapias físicas y los ejercicios de estiramiento muscular. Luego y dependiendo de la respuesta, se considera el tratamiento farmacológico, que como se describió, cuenta con una amplia gama de grupos terapéuticos que incluyen AINEs, opioides, anticonvulsivantes, ansioliticos, antidepresivos y relajantes musculares, fármacos que se deben elegir dependiendo del diagnóstico definitivo, considerando la eficacia mostrada en los diferentes estudios y meta-análisis y teniendo en cuenta las patologías de base del paciente, a fin de minimizar los efectos secundarios y de optimizar la rápida respuesta positiva del enfermo. En todos los casos sin embargo y a la par con las terapias farmacológicas, el profesional tratante no debe olvidar el valor de las terapias de rehabilitación educacional y biopsicosocial, cuando se espera una recuperación integral y sostenida de los pacientes.

\section{Referencias}

1. Navarro C. Tratado de Cirugía Oral y Maxilofacial. 2007; Tomo I. Cap 21

2. Koopman J, Dieleman J P, Huygen F J, De Mos C, Sturkenboom M. Incidence of Facial Pain in the General Population. International Association for the Study of Pain. 2009;147:122-127.

3. Sarlani E, Balciunas B, Grace E. Assessment and Management of Musculoskeletal and Neuropathic Causes. AACN Clinical Issues. 2005;16(3):333-346.

4. Riley J L, Gilbert G. Orofacial Pain Symptoms: An Interaction between Age and Sex. Pain. 2001;90:245-256.

5. Fischer D, Klasser G, Epstein J. Cancer and Orofacial Pain. Oral Maxillofacial. Surgical Clinics of North America. 2008;20:287-301.

6. Moore L. Evaluation of the Patient for Temporomandibular Join Surgery. CAN. 2006; 18 291-303.

7. Okeson J. The Classification of Orofacial Pains. Oral and Maxillofacial Surgery Clinics of North America. 2008;20:133-144.

8. Carlson C R. Psychological Considerations for Chronic Orofacial Pain. Oral and Maxillofacial Surgery Clinics of North America. 2008;20:185-195.

9. Vernon H, Schneider M. Chiropractic Management of Myofascial Trigger Points and Myofascial Pain Syndrome: A Systematic Review Of The Literature. Journal of Manipulative and Physiological Therapeutics Vernon and Schneider. 2009;32(1):14-24.

10. Perez S, Oliván B, Arnal A, Mayoral O. Contributions of Myofascial Pain in Diagnosis and Treatment of Shoulder Pain: A Randomized Control Trial. BMC Musculoskeletal Disorders. 2009;10(92):1-7.

11. Manolopoulos L, Vlastarakos P V, Georgiou L, Giotakis I, Loizos A, Nikolopoulos T. Myofascial Pain Syndromes in the Maxillofacial Area: A Common but Underdiagnosed Cause of Head 
and Neck Pain. International Journal of Oral and Maxillofacial Surgery. 2008;37:975-984.

12. Prithvi P, Paradise L A. Myofascial Pain Syndromeand Its Treatment in Low Back Pain. Seminars in Pain Medicine. 2004;2:167-174.

13. Benoliel R, Birman N, Eliav E, Sharav Y. The International Classification of Headache Disorders: Accurate Diagnosis of Orofacial Pain? Cephalalgia. 2008;28:752-762.

14. Hersh EV, Balasubramaniam R, Pinto A. Pharmacologic Management of Temporomandibular Disorders. Oral and Maxillofacial Surgery Clinics of North America. 2008;20:197-210.

15. Mercuri L G. Osteoarthritis, Osteoarthrosis, and Idiopathic Condylar Resorption. Oral and Maxillofacial Surgery Clinics of North America. 2008;20:169-183.

16. Lewis M A, Sankar V, De Laat A, Benoliel R. Management of Neuropathic Orofacial Pain. Oral Surgery, Oral Medicine, Oral Pathology, Oral Radiology, and Endodontology. 2007;103(1):S32.e1-24.

17. Benoliel R, Eliav E. Neuropathic Orofacial Pain. Oral and Maxillofacial Surgery Clinics of North America. 2008;20:237-254.

18. Nixdorf D R, Velly A M, Alonso A A. Neurovascular Pains: Implications of Migraine for the Oral and Maxillofacial Surgeon. Oral and Maxillofacial Surgery Clinics of North America. 2008;20:221-235

19. Clark G T. Classification, Causation and Treatment of Masticatory Myogenous Pain and Dysfunction. Oral and Maxillofacial Surgery Clinics of North America. 2008;20:145-157.

20. Scrivani S J, Mathews ES, Maciewicz R J. Trigeminal Neuralgia. . Oral Surgery, Oral Medicine, Oral Pathology, Oral Radiology, and Endodontology. 2005;100:527-538.

21. Sato J, Saitoh T, Notani K, Fukuda H, Kaneyama K, Segami N. Diagnostic Significance of Carbamazepine and Trigger Zones in Trigeminal Neuralgia. Oral Surgery Oral Medicine Oral Pathology. 2004;97:18-22.

22. Mounsey A L, Matthew L G, Slawson D C. Herpes Zoster And Postherpetic Neuralgia: Prevention and Management. American Academy of Family Physicians. 2005;72(6):1075-1080.

23. Arias M. ¿Es la Fibromialgia una Enfermedad Neurológica? Servicio de Neurología Complexo Hospitalario Universitario Santiago de Compostela (La Coruña). Neurología. 2008;23(9):593-601.

24. Ubago M C, Ruiz I. Analysis of the Impact of Fibromyalgia on Quality of Life: Associated Factors. Clinical Rheumatology. 2008;27:613-619.

25. Lera S, Gelman S, López M J, Abenoza M, Zorrilla J G, Castro J, Salamero M. Multidisciplinary Treatment of Fibromyalgia: Does Cognitive Behavior Therapy Increase the Response to Treatment? Journal of Psychosomatic Research. 2009;67:433-441.

26. Zapata A L, Pantoja A J. Pain and Musculoskeletal Pain Syndromes in Adolescents. Journal of Adolescent Health. 2006;38:769-771.

27. Pérez-Pareja J, Borrás C, Sesé A. Percepción de Dolor y Fibromialgia. Actas Españolas de Psiquiatria. 2005;33(5):303-310.

28. González J L, Peñacoba C, Velasco L, López A. Mercado F, Barjola P. Recursos Cognitivos de Percepción de Control, Procesos de Estrés/Recuperación y Malestar Afectivo en Fíbromialgia. Psicothema. 2009;21(3):359-368.

29. Cummings M, Baldry P. Regional Myofascial Pain: Diagnosis and Management. Best Practice and Research Clinical Rheumatology. 2007;21(2):367-387.

30. Ruiz M, Nadador V, Fernández J, Hernández J. Dolor de Origen Muscular: Dolor Miofascial y Fibromialgia. Revista de la Sociedad Española del Dolor. 2007;1:36-44.
31. Malchow R J, Black I H. The Evolution of Pain Management in the Critically Ill Trauma Patient: Emerging Concepts from the Global War on Terrorism. Critical Care Medicine. 2008;36(7):346-357.

32. Zuniga J R, Ibanez C, Kozacko M. The Analgesic Efficacy and Safety of Intra-Articular Morphine and Mepivicaine Following Temporomandibular Joint Arthroplasty. Journal of Oral and Maxillofacial Surgery. 2007;65:1477-1485.

33. Hammonds B, Sidebotham D, Anderson B J. Aspects of Tramadol and Ondansetron Interactions. Acute Pain. 2003;5:31-34.

34. Strumpf M, Kulka P, Zenz M. Efficacy and Safety of Tramadol and Morphine in Patients with Extremely Severe Postoperative Pain. Acute Pain. 2000;3(3):1-7.

35. Tuzuner A M, Ucok C, Kucukyavuz Z, Alkis N, Alanoglu Z. Preoperative Diclofenac Sodium and Tramadol for Pain Relief After Bimaxillary Osteotomy. Journal of Oral and Maxillofaccial Surgery. 2007;65:2453-2458

36. Bennett R, Karim K. Tramadol and Acetaminofen Combination Tablets in the Treatment of Fibromyalgia Pain: A Double-blind, Randomized, Placebo-Controlled Study. The American Journal of Medicine. 2003;114:537-545.

37. Pagliara L, Tornago S. Tramadol Compared with Diclofenac in Traumatic Musculoskeletal Pain. Current Therapeutic Research. 1997:58(1):473-480

38. Podolecki T, Podolecki A, Hrycek A. Fibromyalgia: Pathogenetic, Diagnostic and Therapeutic Concerns. Polskie Archiwum Medycyny Wewnetrznej. 2009;119(3):157-161.

39. Willoughby DA, Moore AR, Colville-Nash PR. COX-1, COX-2, and COX-3 and the future treatment of chronic infl ammatory disease. Lancet 2000; 355:646-48.

40. Habib A and Gan T. Role of Analgesic Adjuncts in Postoperative Pain Management. Anesthesiology Clinics of North America. 2005;23:85-107

41. Forbes A B. A Regional Audit of the Use of COX-2 Selective NonSteroidal Anti-Inflammatory Drugs (NSAIDs) in Rheumatology Clinics in the West Midlands, In Relation to NICE Guidelines. Rheumatology. 2005;44:921-924.

42. González-Escalada J R, Rodríguez M J, Camba M A, Portolés A, López R. Recomendaciones para el Tratamiento del Dolor Neuropático. Revista de la Sociedad Española del Dolor. 2009;16(8):445-67.

43. Jung J, Kim D K, Kim M, Kim H J. Onset of Analgesia and Analgesic Efficacy of Tramadol/Acetaminophen and Codeine/ Acetaminophen/Ibuprofen in Acute Postoperative Pain: A Single-Center, Single-Dose, Randomized, Active-Controlled, Parallel-Group Study in a Dental Surgery Pain Model. Clinical Therapeutics. 2004;26(7):1037-1045.

44. Joshia A, Pararab E, Macfarlane T V. A Double-blind Randomized Controlled Clinical trial of the Effect of Preoperative Ibuprofen, Diclofenac, Paracetamol with Codeine and Placebo Tablets for Relief of Postoperative Pain after Removal of Impacted Third Molars. The British Association of Oral and Maxillofacial Surgeons. 2004;42:299-306.

45. Savage M G, Henry M A, Colo D. Preoperative Nonsteroidal Antiinflammatory Agents: Review of the Literature. Oral Surgery, Oral Medicine, Oral Pathology, Oral Radiology, and Endodontology. 2004;98:146-52.

46. Malley P, Balden E, Tomkins G. Treatment of Fibroyalgia with Antidepressants. Journal of General of Internal Medicine. 2000;15:659-666

47. Kurtoglu C, Gur H, Kurkcu M, Sertdemir Y, Guler-Uysal F, Uysal H. Effect of Botulinum Toxin-A in Myofascial Pain Patients with or without Functional Disc Displacement. Journal of Oral and Maxillofacial Surgery. 2008;66:1644-1651. 
48. Lang A M. A Preliminary Comparison of the Efficacy and Tolerability of Botulinum Toxin Serotypes $A$ and $B$ in the Treatment of Myofascial Pain Syndrome: A Retrospective. Open-Label Chart Review, Clinical Therapeutics. 2003;25(8):2268-2278.

49. Gobel H, Heinze A, Reichel G, Hefter H, Benecke R. Efficacy and Safety of a Single Botulinum Type A Toxin Complex Treatment (Dysport) for the Relief of Upper Back Myofascial Pain Syndrome: Results from a Randomized, Results from a randomized double-blind placebo-controlled multicentre study. Pain. 2006;125:82-88.

50. Balasubramaniam R, Ram S. Orofacial Movement Disorders. Oral and Maxillofacial Surgery Clinics of North America. 2008;20:273-285.
51. Jeffrey P O. Management of Temporomandibular Disorders and Occlusion. Mosby. 2008; $6^{\circ}$ edición.

52. Katzung B G. Farmacología Basica y Clinica. 2007. 10 edicion.

53. Gonima E. Guías de Manejo del Dolor. Hospital Militar Central. 2009.

54. Phero J C, Becker D E, Dionne R A. Contemporary Trends in Acute Pain Management. Current Opinion in Otolaryngology and Head and Neck Surgery. 2004;12:209-216.

55. Prasad A, Galetta S. Trigeminal Neuralgia Historical Notes and Current Concepts. The Neurologist. 2009;15:87-94. 